\title{
BANDANA - Body Area Network Device-to-device Authentication using Natural gAit
}

\author{
(Extended Version of PerCom 2017 Submission) \\ Dominik Schürmann*, Arne Brüsch* ${ }^{*}$, Stephan Sigg ${ }^{\dagger}$ and Lars Wolf* \\ *Institute of Operating Systems and Computer Networks, TU Braunschweig \\ Email: \{schuermann, bruesch, wolf $\} @$ ibr.cs.tu-bs.de \\ ${ }^{\dagger}$ Ambient Intelligence, Comnet, Aalto University \\ Email: \{arne.bruesch, stephan.sigg $\} @$ aalto.fi
}

\begin{abstract}
Secure spontaneous authentication between devices worn at arbitrary location on the same body is a challenging, yet unsolved problem. We propose BANDANA, the first-ever implicit secure device-to-device authentication scheme for devices worn on the same body. Our approach leverages instantaneous variation in acceleration patterns from gait sequences to extract alwaysfresh secure secrets. It enables secure spontaneous pairing of devices worn on the same body or interacted with. The method is robust against noise in sensor readings and active attackers. We demonstrate the robustness of BANDANA on two gait datasets and discuss the discriminability of intra- and inter-body cases, robustness to statistical bias, as well as possible attack scenarios.
\end{abstract}

\section{INTRODUCTION}

Scalable secure device pairing in the wake of the Internet of Things (IoT) is a pending problem that has not yet been solved satisfactorily. Current pairing protocols include pinbased approaches (e.g. Bluetooth) or out-of-band communication [1]. Example out-of-band channels are, for instance, a secret printed on or displayed by a device, Near Field Communication [2] or audie [3]. Alternatively, the standard approach for IoT security is to have the device connect to a dedicated trusted server which then handles the pairing and necessary key-exchange of the devices in question [4].

These approaches are sufficient for one-time manual pairing of a limited number of devices. However, the personal devicenetwork in the IoT is expected to experience frequent fluctuation in device count and identity [5]. New devices are added in the context of use while others are discarded. Examples are pairings to a multitude of body-worn smart devices (watches, glasses, smartphone, bio-sensors), fitness trackers, changing smart textile, or to temporarily used external devices such as bicycles, cars, shopping carts, or equipment in a fitness center. While seamless pairing without manual user interaction among such devices promises new, personalised services, the threat of privacy exposion to malicious adversaries needs to be controlled by novel secure pairing schemes that scale.

As depicted in Figure 1, we envision spontaneous secure pairing which allows frequent re-pairing (restricted to the timeof-use), and ad-hoc implicit (no manual interaction required)

\footnotetext{
${ }^{1} \mathrm{~A}$ popular commercially implemented example using audio to initiate device pairing is the chromecast protocol
}

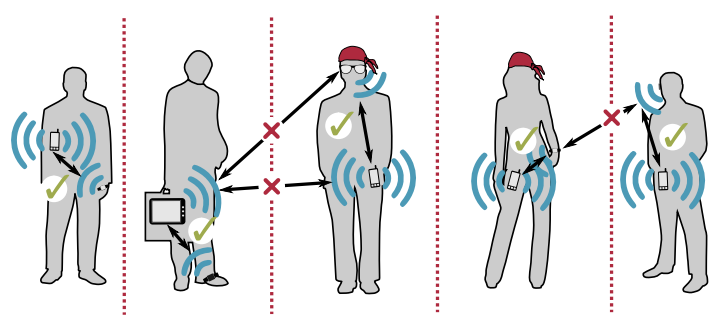

Fig. 1: BANDANA creates implicit security barriers towards devices in proximity, while establishing ad-hoc spontaneously secure connections between devices worn on the same body.

secure authentication bound to an individual. The desired solution shall not require a trusted third party.

This paper proposes a solution to this challenge by introducing a secure pairing scheme among on-body devices based on common movement patterns due to co-location on the same body. In particular, we exploit instantaneous variations in gait sequences for implicit generation of a shared secret among all devices on the same body. The contributions of our work are

1) a secure ad-hoc pairing scheme for devices worn on the same body

2) the experimental verification of the protocol on a number of large-scale gait datasets

3) security analysis on the pairing approach covering Entropy, statistical bias, and attack scenarios

The remainder of the paper is organized as follows. In section [I] we introduce previous studies on ad-hoc secure device pairing and how they relate to our work. We will then in section III introduce basic methodology on gait cycle detection, datasets utilised in our evaluation, and data preparation, before introducing our secure ad-hoc pairing scheme, BANDANA in section IV] In section VI attack models on the protocol are discussed and in section $[\mathrm{V}$, the proposed adhoc pairing based on instantaneous gait-patterns is evaluated on a number of large-scale gait datasets and with respect to Entropy, statistical bias, as well as random and sophisticated attacks. Finally, section VII concludes our discussion. 


\section{RELATED WORK}

A popular sensor to detect co-presence is the accelerometer. For instance, [6] present a process to generate shared keys based on shaking processes. They propose a threshold-based protocol conditioned on the magnitude of the co-aligned acceleration processes. A similar approach has been followed by Mayrhofer et al. [7], who demonstrated that an authentication is possible when devices are shaken simultaneously by a single person, while an authentication was unlikely for a third person trying to mimic the correct movement pattern remotely. Improvements to this protocol have been proposed, for instance, in [8], where the success probability of devices in proximity is increased by considering acceleration patterns derived in the order of their magnitude. Also, [9] solves the issue of different sample rates on paired devices and nonaligned starting points while [10] mitigate relative differences in rotation among devices. Based on this protocol, [11, 12] presents an approach to unlock a mobile device with the help of a smartwatch when both are shaken simultaneously. Their approach, however, requires that acceleration sequences are exchanged and compared via an established secure channel. Another implementation was presented in [13] to authenticate from the acceleration pattern of a vibrating device lying on a flat surface. Applications for such schemes have been proposed, for instance, in [14] where devices connected via NFC are authenticated via acceleration traces of vibrating smart phones that touch each other. Also, [15] propose to prevent man-in-the-middle relay attacks on NFC payment by requiring matching acceleration sequences generated during double-tapping one device on the other [16]

For authentication based on arbitrary co-aligned sensor data, the candidate key protocol is proposed in [17]. It interactively exchanges hashes from feature sequences as short secrets and concatenates the key from the secrets with matching hashes.

Examples for possible sensor modalities that can be used for unattended co-presence-based device pairing apart of acceleration are magnetometer [18], RF-signals [19, 20] luminosity [21] or audio [3]. These, however, have in common that the pairing is not constrained to devices on the same body but, more generally, to devices in proximity.

We are, in contrast, focusing on the pairing of devices on the same body or interacted with by the same person. Consider, for instance, [22], where key-card authentication is extended with additional acceleration patterns.Another example is presented in [23] to authenticate a user wearing an acceleration-capturing bracelet during interaction with a keyboard device. The device is locked when keyboard-interaction and acceleration patterns mismatch.

Very related to our study is the work of Cornelius et al. [24] to identify devices co-located on the same body via correlated acceleration readings. An important problem to consider when comparing acceleration sequences among devices on the body is that orientation and placement significantly impact the recorded acceleration, gyro and magnetometer sequences [25]. Solutions to receive placement independent features are, (A) to calculate the norm or magnitude $m_{i}=\sqrt{x_{i}^{2}+y_{i}^{2}+z_{i}^{2}}$ (thereby discarding information on acceleration along individual axes [26]), (B) to first detect the location on the body and then to try to deal with changes that occur due to placement [25], or (C) to tackle disorientation and misplacement errors by calculating the rotation matrix from magnetometer readings [27]. Even though after (A), the resulting signal still differed greatly due to inherently differing movement of underlying body parts (e.g. arm vs. head vs. legs) [28], Cornelius et al. [24] succeeded to show good correlation among all body locations from mean, standard deviation, variance, mean absolute deviation and interquartile range as well as signal's energy. This result is a strong indication that secure keys conditioned on co-location on the same body exist. However, as correlation can be alternating positively and negatively, it remains unsolved how this can be exploited for the generation of keys, when the sequences shall not be disclosed to an adversary listening to any communication between nodes.

A weakness of using arbitrary acceleration sequences for spontaneous pairing of on-body devices is that for a significant number of daily activities, upper body and lower body movements are only weakly or not correlated. An activity that can be well recognized over the whole body though is walking or gait [29]. For instance, identical step patterns from acceleration sequences have been utilised for co-location detection [30]. The authors in [31, 32] employ gait cycles to authenticate a user on his smart-phone by matching the current walking pattern against a previously saved walking template exploiting a fuzzy commitment scheme [33]. Another gait-based authentication system is proposed in [34] where a two-stage classifier first distinguishes walking from other activity to then exploit individual gait patterns. A concern for any authentication scheme based on implicit features is that the generated key sequences employ a high randomness and are uniformly distributed over the key space so that an adversary can not easily guess or re-produce the key. To this end, [35] recently presented an approach to generate a key fingerprint from the difference of a mean world gait (spanning the complete population) to the mean gait of an individual. By computing the mean gait over the whole population, the authors assured that the resulting sequence is well balanced and uniformly distributed.

These studies on gait-based authentication (1) do not address the impact of different on-body locations and sensor orientation and (2) intend to use gait as a unique biometric feature that does not change for an individual over time. In contrast, in our case, we intend to generate an always-fresh authentication key based on instantaneous acceleration sequences for arbitrary location on the human body. Muaaz et al. [36] confirmed the significant challenge of (1) but demonstrated gait-based authentication covering closely related locations on the human body (from one to the other side of the hip) is possible but suffers from high error rate.

For the verification and security analysis of gait-based authentication schemes, it is crucial to test the approach on 


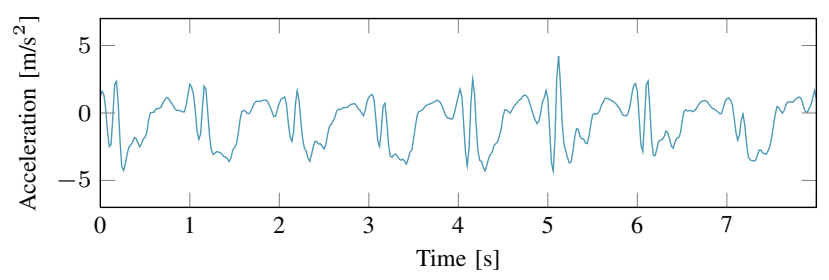

(a) Unmodified accelerometer reading (z-axis) at $50 \mathrm{~Hz}$.

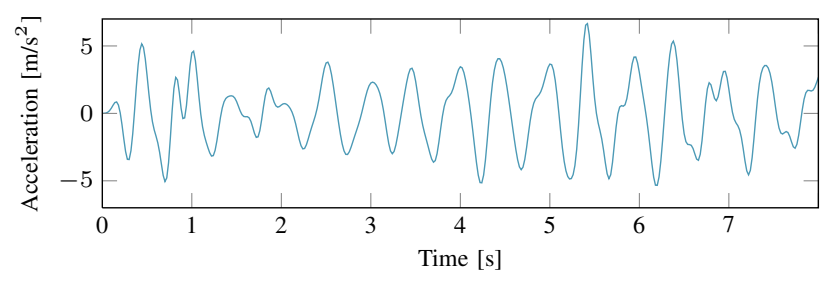

(c) Application of Type-II Chebyshev bandpass filter.

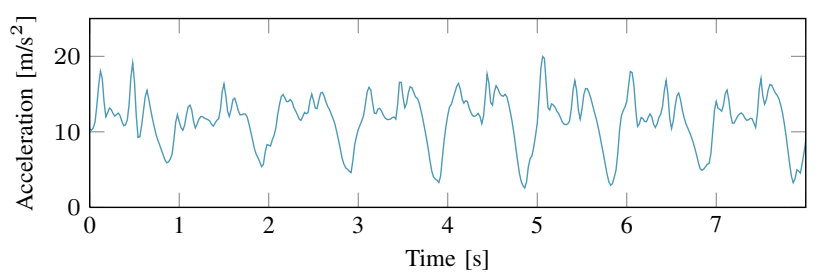

(b) Application of Madgwick's algorithm. It should be noted that the gravity $g=\sim 9.81 \mathrm{~m} / \mathrm{s}^{2}$ can now be recognized, indicating a correct orientation relative to the ground.

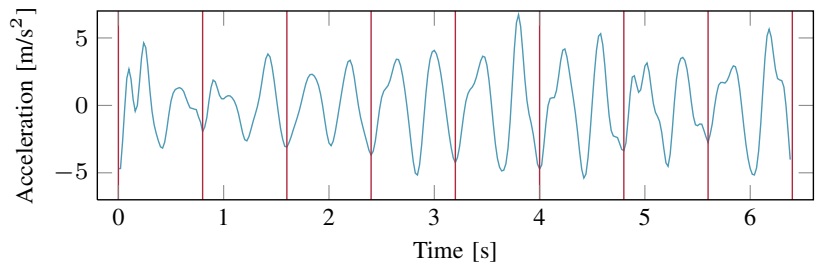

(d) Resampling to $\rho=40$ and Gait Cycle Detection with $q=8$ gait cycles.

Fig. 2: Example of our process for sensor data pre-processing and gait cycle detection. Here, the z-axis of an accelerometer is depicted, which is attached to the forearm of one subject.

a large number of participants. To this end, [37] present a gait dataset for 100 participants wearing accelerometers at the hip. Another dataset with 744 subjects has been presented in [38]. Participants traverse a parcours featuring distinct walking conditions such as straight, coarse ground, upwards, downwards and steps. Also, Sztyler et al. created a real-world dataset used for Position Aware Activity Recognition [39] in which 15 subjects, equipped with sensors on 7 distinct locations on the body performed different actions for a time period of approximately 10-12 minutes each.

Another, conceptional challenge with all context-based authentication approaches is that due to sensing inaccuracies, different hardware and noise the sensed signals are likely not identical but only similar. Fuzzy cryptography presents a methodology to obtain identical keys from similar patterns [33]. In particular, by mapping the patterns into the codespace of an error correcting code, mismatches can be mitigated without disclosing the pattern over a potentially insecure channel.

\section{FUNDAMENTALS}

\section{A. Gait Cycle Detection}

In this section, our gait cycle detection algorithm is presented which builds on ideas by Hoang et al.[31, 32]. In addition, we also utilize gyroscope readings to normalize the sensor's orientation and keep only the z-Axis that points in the opposite direction of gravity.

As depicted in Figure 3, a gait cycle is defined as the "time interval between two successive steps", e.g., if a cycle starts with the initial contact of the right foot, it ends with the same position of the right foot [41]. Thus, the goal of the algorithm
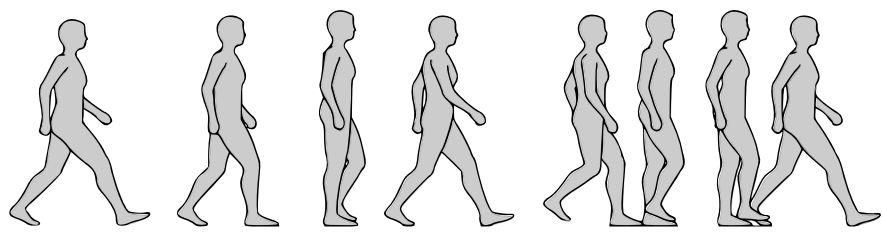

Fig. 3: A full gait cycle starting with the initial contact of the right foot. Characteristic intermediate positions are depicted separately. (modified from [40])

is to find repetitive cycles in the raw acceleration signal.Its input is a vector of amplitude values

$$
\boldsymbol{z}=\left(z_{1}, \ldots, z_{n}\right)
$$

of the accelerometer z-axis (cf. Figure 2a).Its output is a gait sequence of consecutive gait cycles with normalized length.

To find repetitive parts in the signal, a naive approach would search for local minima and then use these to split the raw signal into cycles. However, the list of all local minima (comparing only direct neighbors) contains too many minima within single gait cycles. Thus, this list must be filtered for minima having the same distance to each other to define clearly separated cycles.

Our filtering method is based on autocorrelation and distance calculation. The discrete autocorrelation at time lag $k$ and with variance $\sigma^{2}$ is estimated as

$$
\operatorname{Acorr}(k)=\frac{1}{(n-k) \sigma^{2}} \sum_{t \in \mathbb{Z}} z_{t+k} \cdot \bar{z}_{t}
$$

where $\bar{z}_{t}$ represents the conjugate of $z_{t}$. The resulting autocorrelation

$$
\boldsymbol{a}=\left(a_{1}, \ldots, a_{n}\right)
$$


Mannheim: The Real-World Dataset by Sztyler et al. was used for position aware activity recognition [39]. 15 Subjects performed different actions for a time period of approximately $10-12$ minutes each. They were equipped with 7 sensors on different parts of the body. These parts were chosen in order to gather data from every part of the body that acts different during human action.

Osaka: The OU-ISIR Gait Database [38] consists of samples taken from three triaxial accelerometers and gyroscopes worn on different parts of the thigh (left, right, center). With this setup, subjects were asked to walk down a path, upstairs and down a slope. A total of 460 records exist within this dataset.

Fig. 4: Datasets utilised in our study

leads to $m$ non-ambiguous local maxima in $\boldsymbol{a}$, stored as

$$
\zeta=\left\{\zeta_{1}, \ldots, \zeta_{i}, \ldots \zeta_{m}\right\} .
$$

The distances between these indices and a mean distance

$$
\delta_{\text {mean }}=\left\lceil\frac{\sum_{i=1}^{m-1} \zeta_{i+1}-\zeta_{i}}{m-1}\right\rceil
$$

are calculated. $\delta_{\text {mean }}$ defines the length of half a cycle, i.e., the time between the initial contact of the starting foot followed by the initial contact of the subsequent foot. Thus, for $q$ describing the number of gait cycles, $m=q \cdot 2$. For the gait-cycle extraction, we assume healthy subjects, where the movement of the right foot is sufficiently similar to the left foot and thus have nearly the same distance. $\delta_{\text {mean }}$ can now be used to select indices of minima from $z$ that represent clear cycles with the same length:

$$
\begin{aligned}
\boldsymbol{\mu} & =\left\{\mu_{1}, \ldots, \mu_{i}, \ldots, \mu_{m-1}\right\} \\
\mu_{i} & =\arg \min \left(z_{\zeta_{i}-\tau}, z_{\zeta_{i}-\tau+1}, \ldots, z_{\zeta_{i}+\delta_{\text {mean }}+\tau}\right) .
\end{aligned}
$$

Every $\mu_{j}$ represents the index of a minimum in $z$ limited to the range of $\delta_{\text {mean }}$ where $\tau$ defines an additional user defined factor to account for small deviations in the gait duration. The indices in $\boldsymbol{\mu}$ can now be used to split the raw data $\boldsymbol{z}$ into full gait cycles

$$
\begin{aligned}
Z & =\left\{Z_{1}, \ldots, Z_{i}, \ldots, Z_{q}\right\} \\
Z_{i} & =\left(z_{\mu_{\frac{i}{2}}}, \ldots, z_{\mu_{i}}, \ldots, z_{\frac{\mu_{\frac{i+1}{2}}}{2}-1}\right) \\
i & =\{2,4, \ldots, q\}
\end{aligned}
$$

Finally, the length of gait cycles are normalized by resampling every $Z_{i}$ using a Fourier method to a fixed number of samples $\rho$ per gait cycle so that $\left|Z_{i}\right|=\rho$ (cf. Figure 2d). For ease of presentation, we will, in the following, describe such normalized gait cycle with $Z_{i}=\left\{Z_{i 1}, \ldots, Z_{i \rho}\right\}$. The choice of $\rho$ depends on factors such as sample rate and requirements of the quantization algorithm discussed in Section IV.

\section{B. Datasets}

In our work, we used two different datasets gathered for evaluation of human movement (cf. Figure 4). We refer to

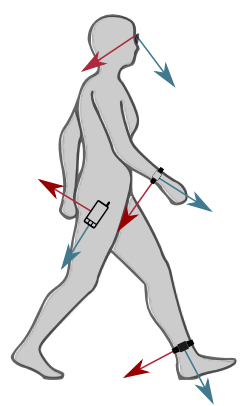

(a) Before

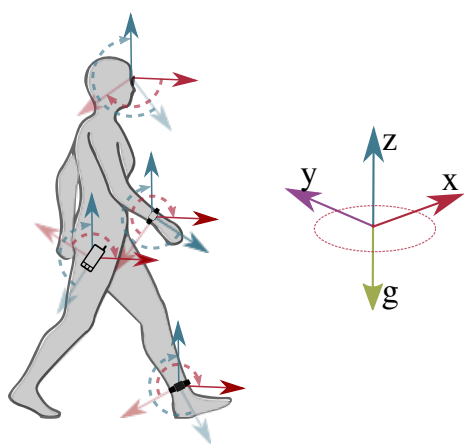

(b) After Madgwicks algorithm
Fig. 5: Different Sensors worn on a human body. Depicted are the devices' coordinate systems before and after application of Madgwicks algorithms. Note the remaining degree of freedom along the xy-plane.

the datasets as the osaka respectively the mannheim dataset in accordance with the hometown of their collectors. Unfortunately, the osaka dataset has certain faults.A conceptual issue lies in the fact that all sensor units were located on pretty close positions on the body. As well, they were mounted to the same harness, introducing a possible error. Finally, the osaka dataset yields only 6-8 Gait Cycles of stationary walk per subject.

\section{Data Pre-Processing}

In a real-world setting, it is very likely that different sensor platforms are worn at different positions, which introduce dynamically changing orientations due to body part movements (cf. Figure 5a). For our gait cycle detection to work well, it is crucial to align these every data point of these different orientations such that one of the axes is facing in the opposite direction of gravity as depicted in Figure $5 \mathrm{~b}$

Nowadays, most mobile devices contain gyroscopes in addition to accelerometers [42]. We therefore posses information about the initial device orientation (since the force of gravity is included in every measurement recorded by the accelerometer) as well as the angular velocity of the sensor platform itself. Thus, it is possible to correct the ongoing orientation error. We employ the algorithm proposed by Madgwick et al. [43] to rotate all measurements $z_{i}$ accordingly, resulting in a signal as shown in Figure 2b. Note that the output is only guaranteed to be aligned along the z-axis which is in parallel with the gravity axis. When comparing two readings, both other axes may point in different directions because no second fixed direction as, for example, the direction of North is obtainable.

Madgwick's algorithm only changes the sensor orientation (see Figure 5), it does not remove any noise. Therefore, we apply a Type II Chebyshev bandpass filter with passband chosen between $0.5 \mathrm{~Hz}$ and $12 \mathrm{~Hz}$. Cutoff frequencies are further discussed in section $\mathrm{V}-\mathrm{B}$. The resulting signal is shown in Figure 2c 

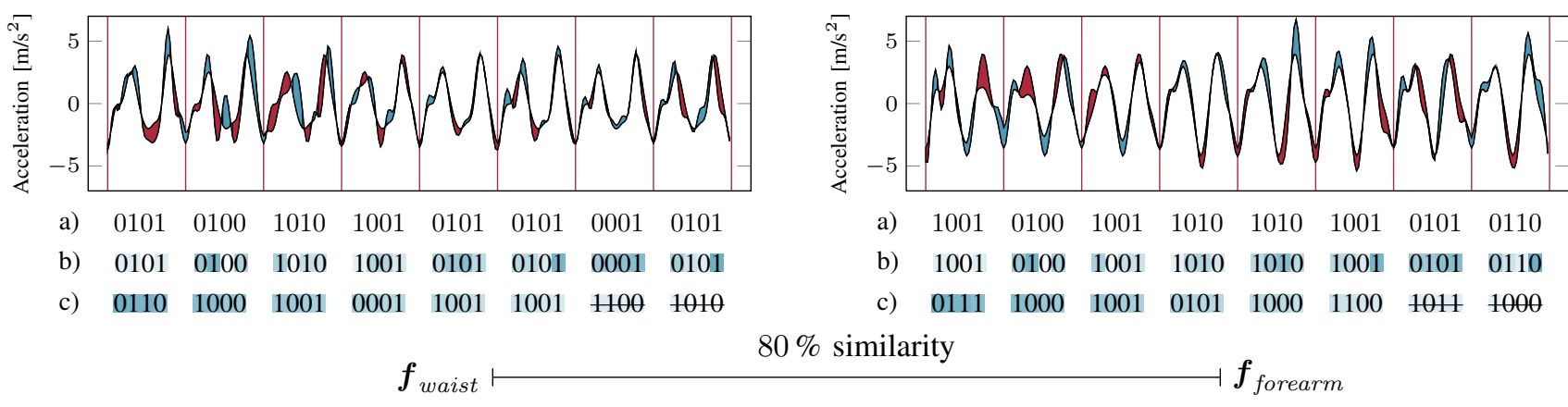

Fig. 6: Independent fingerprint generation on sensor positions waist and forearm: The graph shows energy levels above the average gait cycle $\boldsymbol{A}$ as blue and below as red areas. After quantization in (a), reliabilities are calculated and assigned to each bit in (b). The darker the color, the more reliable the bit. Finally, in (c) the fingerprint is sorted by reliability vector of the forearm with a cutoff at 24 .

\section{BANDANA}

After recording raw accelerometer as well as gyroscope data and correcting sensor-orientations together with applying a band-pass filter, the gait cycle detection algorithm produces a periodic filtered signal. For BANDANA's device-to-device authentication, shared secrets need to be generated based on these signals independently on participating devices and, in particular, without disclosing information on the gait sequence on the communication channel.

\section{A. Quantization}

To generate binary fingerprints from the continuous gait sequence, we propose a quantization algorithm inspired by Hoang et al. [31]. Recall the definition of $Z_{i}$ from equation (1) with the normalized gait cycle $\left|Z_{i}\right|=\rho$ and $Z_{i}=$ $\left\{Z_{i 1}, \ldots, Z_{i \rho}\right\}$. We define the average gait cycle as

$$
\begin{aligned}
\boldsymbol{A} & =\left(A_{1}, \ldots, A_{j}, \ldots A_{\rho}\right) \\
A_{j} & =\frac{\sum_{i=1}^{q} Z_{i j}}{q} .
\end{aligned}
$$

Fingerprint bits are extracted by calculating the energy difference between each gait cycle $Z_{i}$ and $\boldsymbol{A}$ as depicted in Figure 6 . To extract $b$ bit per $Z_{i}$, each $Z_{i}$ is split into $b$ parts of the same length $\rho / b$. Thus, a binary fingerprint is defined by

$$
\begin{aligned}
\tilde{f} & =\left(\tilde{f}_{11}, \ldots, \tilde{f}_{1 \frac{\rho}{b}}, \ldots, \tilde{f}_{b 1}, \ldots, \tilde{f}_{b \frac{\rho}{b}}\right) \\
\tilde{f}_{i j} & = \begin{cases}1, & \delta_{i j}>0 \\
0, & \text { otherwise. }\end{cases} \\
\delta_{i j} & =\sum_{k=0}^{\rho / b} A_{i+k}-Z_{i+k, j} .
\end{aligned}
$$

as exemplary shown in Figure 6 (a). In the following, the fingerprint vector is written as

$$
\tilde{\boldsymbol{f}}=\left(\tilde{f}_{1}, \ldots, \tilde{f}_{M}\right) .
$$

\section{B. Reliability}

To calculate the reliability of the extracted bits, the differences of the quantization algorithm are stored as

$$
\boldsymbol{\delta}=\left(\delta_{11}, \ldots, \delta_{1 b}, \ldots, \delta_{q 1}, \ldots, \delta_{q b}\right) .
$$

The indices of $\delta$ are sorted in descending order by the absolute value of each associated difference $\left|\delta_{i j}\right|$ to retrieve the reliability ordering

$$
\boldsymbol{r}=\left(r_{1}, \ldots, r_{M}\right) \text { with } r_{i} \geq r_{i+1} .
$$

We will refer to $r$ in the following as the reliability vector. It contains those indices which experienced the highest difference between the mean gait cycle $\boldsymbol{A}$ an an instantaneous normalized gait cycle $Z_{j}$. These bits are most reliable in the sense that they have high probability to be identical for devices at arbitrary body positions. In Figure 6 (b) we used colors to indicate the associated reliability. The elements of $\tilde{f}$ are then sorted according to their corresponding values of $\boldsymbol{r}$ and the most reliable first $N$ constitute the final fingerprint $\boldsymbol{f}=$ $\left(f_{r_{1}}, \ldots, f_{r_{N}}\right)$ (cf. Figure 6 (c)).

\section{Fuzzy Cryptography}

To derive unique shared secrets on both participating devices without exchanging additional information for comparison, error correcting codes are utilized. Error correcting codes are normally used to encode messages from the messagespace $m \in \mathcal{M}$ into codewords of the (larger) codespace $c \in \mathcal{C}$ introducing redundancies.

$$
m \stackrel{\text { Encode }}{\longrightarrow} c .
$$

This process allows to correct errors introduced when transmitting $c$ over a lossy channel before decoding it back to $m$ with

$$
c \stackrel{\text { Decode }}{\longrightarrow} m \text {. }
$$

We apply error correcting codes in a different way. In a sense, our fingerprints $f$ are lossy as they are not entirely equal on the devices trying to mutually authenticate. Here, the codespace $\mathcal{C}$ is chosen in a way that we can directly pick 
a fingerprint $f$ from this codespace and apply the Decodemethod with

$$
\boldsymbol{f} \stackrel{\text { Decode }}{\longrightarrow} \boldsymbol{k}
$$

to derive a binary key $\boldsymbol{k}$ that is error corrected. Due to the usage of binary fingerprints we propose the usage of BCH codes over the Galois field $\mathbb{F}_{2}$. A BCH code can be parameterized to correct up to $t$ errors, which in our case must be chosen carefully to allow for errors within different positions on the same body but not for correction of errors between different bodies. As with the other parameters, $t$ is chosen based on our evaluation in Section $\mathrm{V}$

\section{Protocol}

Finally, we introduce BANDANA's full protocol flow between two devices $A$ and $B$ worn on the same body. Following Figure 77, the gait cycle detection is applied on recorded accelerometer data corrected by Madgwick's algorithm and Type-II Chebyshev bandpass filter. For two co-aligned devices A (Alice) and $\mathrm{B}$ (Bob), fingerprints $\boldsymbol{f}_{\boldsymbol{A}}\left(\boldsymbol{f}_{\boldsymbol{B}}\right)$ and reliability vectors $r_{A}\left(r_{B}\right)$ are derived on both devices independently. To utilize the same vector for reliability ordering on both sides, a random value $x_{A}\left(x_{B}\right)$ is transmitted together with $\boldsymbol{r}_{A}\left(\boldsymbol{r}_{B}\right)$. The same reliability vector $\boldsymbol{r}$ is then used to sort $\boldsymbol{r}_{\boldsymbol{A}}$ and $\boldsymbol{r}_{B}$. To account for errors, we apply the BCH decodingmethod to reduce both $r_{A}$ and $r_{B}$ to a unique $k$, which is then used as the password for a Password-Authenticated Key Agreement (PAKE). Both devices now share the same secret $s$ protected by a key agreement authenticated by their gait fingerprints. We propose the usage of a modern nonpatented PAKE that feature additional countermeasures for low entropy passwords, such as Password Authenticated Key Exchange by Juggling (J-PAKE) [44] or Secure Remote Password protocol (SRP) [45].

For devices with high clock drift, the protocol can be extended to allow for multiple tries with different fingerprints. For this to work, before quantization the gait sequence should be shifted by half-gait cycles to the right by both devices $A$ and $B$.

\section{Evaluation}

In this section, we present the evaluation of our approach as well as experiments that led us to choose certain parameter configurations.

\section{A. Signal Coherence}

After applying Madgwick's algorithm (cf. section III-C), we end up with sensor readings where the z-axis has been aligned to point to the ground. This allows to examine the relation between these sensor readings. For this, we calculate the spectral coherence for different sensor combinations to test whether any causality between readings taken simultaneously by sensors located in different positions on the same body exists - apart from just the correlation for the respective motion in general. Figure 8 clearly shows that there is hardly any similarity between arbitrary recordings, but a considerably

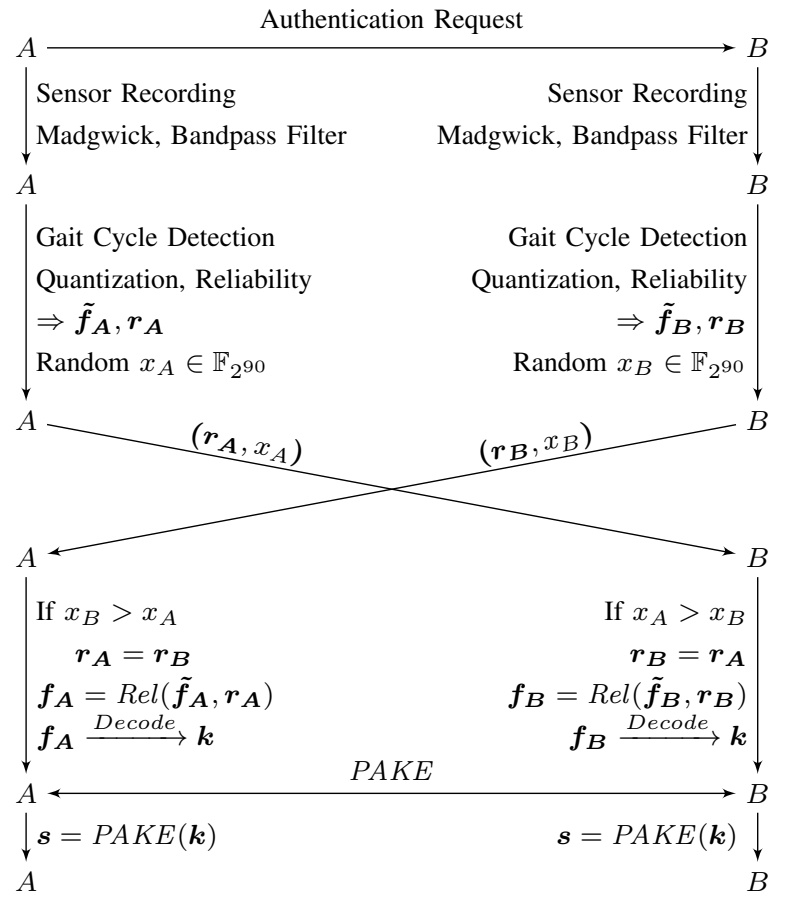

Fig. 7: BANDANA protocol sequence between two devices $A$ and $B$ worn on the same body.

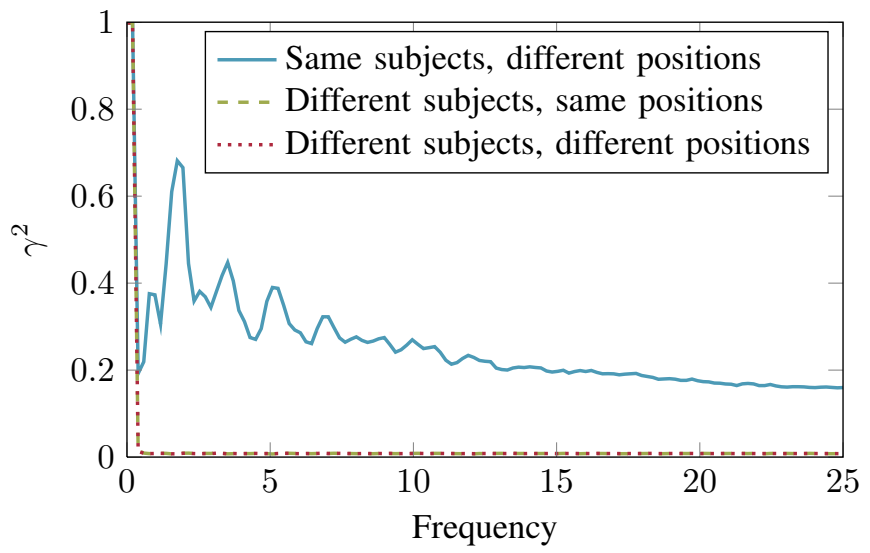

Fig. 8: Average spectral coherence over full sensor readings of the Mannheim dataset for same and different subject.

high correlation between those records being taken simultaneously. Only between $0 \mathrm{~Hz}$ up to $0.5 \mathrm{~Hz}$, a correlation between arbitrary records exists. This leaves us with two major results:

- There is a measurable causality between sensor readings taken simultaneously on the same body

- Some unwanted correlation at lower frequencies still exists

\section{B. Bandpass Filter}

Following the results from the previous section, we continue filtering the sensor readings. As visualized in Figure 8 , there still exists some unexpected correlation between arbitrary 
readings on low frequencies. As these frequencies - up to approximately $0.5 \mathrm{~Hz}$ - only add noise, we would like to filter them out while keeping all the frequencies above. We thus employ a Type-II Chebyshev filter, which is known to have a very steep drop at the cutoff frequency. Furthermore, in contrast to Type-I, Type-II Chebyshev filters do not have any ripple in the passband.

Researchers in the domain of Activity Recognition report that human motion does not affect any frequencies significantly above $10 \mathrm{~Hz}$ [46]. Based on this observation and the coherence depicted in Figure 8 , we decided to choose an upper cutoff frequency of $12 \mathrm{~Hz}$.

\section{Reliability}

Our quantization scheme defines that iff $\delta_{i j}>0$ for fixed $i, j$ is true for Alice, the same has to apply for Bob for at least $80 \%$. Some $Z_{i j}$ are less prone to leading to different bits between sensors at different body locations than others, namely those with a higher difference $\delta_{i j}$ to the mean gait $\boldsymbol{A}$. Both Alice and Bob keep a reliability value for each bit of the fingerprint. According to the BANDANA-Protocol as shown in Figure 7, one of these reliability vectors is chosen randomly and the fingerprint is sorted by each party following tis reliability vector's order of indices (see Figure 6). In a last step, the fingerprint's most unreliable bits are discarded. To show the viability of this approach, we calculated the fingerprints' similarity over all 15 subjects and all 7 sensor positions. As shown in Figure 9, we chose different fingerprint sizes $M$ with cutoff at $N=128$ to test how many additional bits should be discarded by BANDANA to gain the best similarity. The mean-similarity improves with greater values of $M$ and settles around $N+64$ with an average improvement of approximately $4 \%$. Thus, we chose $N+64$ for our configuration.

\section{Discriminability of Intra- and Inter-body Fingerprints}

Figure 10 illustrates the discriminability between intrabody and inter-body fingerprints. The intra-body case with $M=N+64$ with $N=128$ is compared against the inter-body cases of different sensor positions. Here, we evaluated whether similarities exist between different subjects but same sensor positions. While the intra-body case tests only similarities between differing sensor position on the same body (315 similarities), the inter-body case is much larger. Each interbody position case contains 8880300 similarities. As expected, the mean similarity between different subjects is $50 \%$. It is important to note that this test evaluates the worst case of brute forcing all possible combinations between subjects. In reality, an attacker is constrained to $\sim 432$ tries per day (cf. Section VI. In the inter-body case, it can be seen that a small number of fingerprints match with unexpected high similarity values (outliers). We assume that these collisions happen in case of gait sequences with very low entropy still exhibiting specific pattern due to the design of the quantization scheme. While this should be investigated further, only $0.0642 \%$ of these collisions show similarity values above $80 \%$.

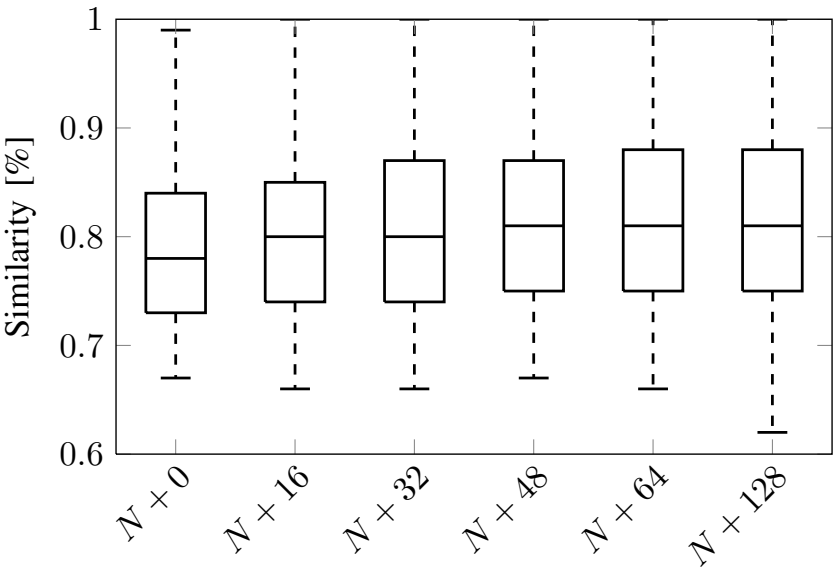

Total Fingerprint Size $M$

Fig. 9: Different fingerprint sizes $M$ with cutoff at $N=128$ to evaluate the influence of $\operatorname{Rel}()$ on intra-body similarity. Each value in this graph is defined by the similarity of two differing sensor positions within the same subject (intrabody). The graph contains all possible similarities within each subject and all her sensor position-combinations. Fingerprints are generated by a sliding window over the sensor data with half-overlapping windows. Only fingerprints generated from the same windows are matched against each other.

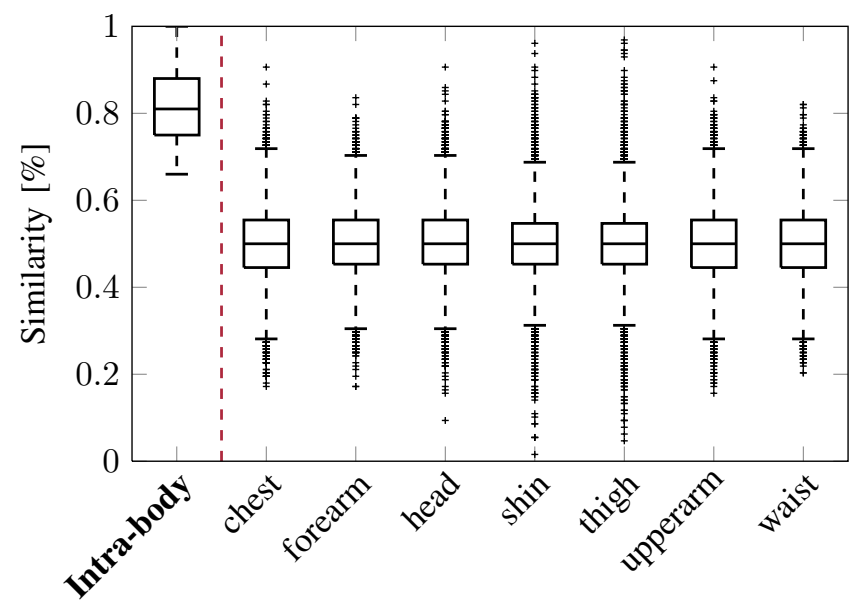

Inter-body

Fig. 10: Comparison of intra-body against inter-body similarity. Each value in the intra-body boxplot is defined by the similarity of two different sensor positions within the same subject (all possible similarities within each subject and all her sensor position-combinations). For the inter-body test, each boxplot defines a different sensor position. Only different subjects are tested against each other with the same sensor positions. Fingerprints are generated by a sliding window over the sensor data with half-overlapping windows for $M=192$ with cutoff at $N=128$. 
TABLE I: Detailed comparison of sensor position-combinations worn on the same body (intra-body). Shown is the mean over all 15 subjects.

\begin{tabular}{|c|c|c|c|c|c|c|c|}
\hline chest & 1.0 & 0.82 & 0.74 & 0.78 & 0.78 & 0.88 & 0.81 \\
\hline forearm & 0.82 & 1.0 & 0.8 & 0.81 & 0.88 & 0.89 & 0.89 \\
\hline head & 0.74 & 0.8 & 1.0 & 0.8 & 0.76 & 0.77 & 0.78 \\
\hline shin & 0.78 & 0.81 & 0.8 & 1.0 & 0.77 & 0.78 & 0.8 \\
\hline thigh & 0.78 & 0.88 & 0.76 & 0.77 & 1.0 & 0.85 & 0.84 \\
\hline upperarm & 0.88 & 0.89 & 0.77 & 0.78 & 0.85 & 1.0 & 0.88 \\
\hline waist & 0.81 & 0.89 & 0.78 & 0.8 & 0.84 & 0.88 & 1.0 \\
\hline
\end{tabular}

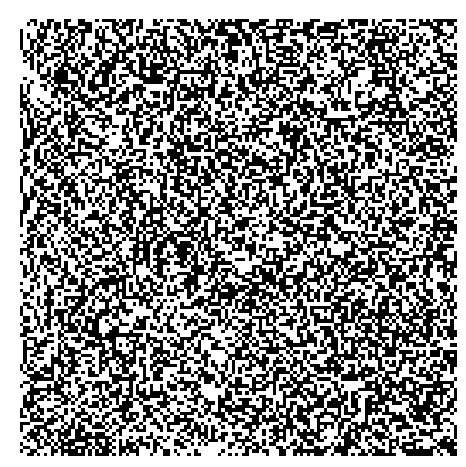

Fig. 11: Illustration of 128 binary keys after removing 64 unreliable bits from the fingerprint. Each row contains one 128 bit fingerprint with $1=$ black and $0=$ white.

\section{E. Similarities between Sensor Position-Combinations}

Table I illustrates how well differing sensor positions authenticate against each other. We found out that chest against other positions and head against other positions perform worse while forearm and waist perform best.

\section{F. Statistical Bias}

For the robustness against a potent adversary, it is important that the keys generated from gait sequences are random. For instance, Figure 11 exemplarily depicts 128 keys we extracted using BANDANA with fingerprint length $M=192$ bits after removing 64 unreliable bits (cutoff at $N=128$ bits) for an intuitive illustration of the randomness of the generated fingerprints.

We rigorously tested the keys generated by BANDANA against statistical bias and employed the dieHarder battery of statistical tests for this end [47]. While these tests can not replace cryptanalysis they are designed to uncover bias and dependency in the pseudo random sequence. Every test has an expected distribution of outcomes. Test runs produce a value that is compared to the theoretical outcome. A pvalue, describing the probability that a real Random Number Generator (RNG) would produce this outcome, between 0 and 1 is computed. A good RNG will have a range of p-values that follows a uniform distribution. A p-value below a fixed significance level $\alpha=0.001$ indicates a failure of the PRNG with probability $1-\alpha$. For instance, a p-value $\leq 0.05$ is exptected $5 \%$ of the time.

Our results are depicted in Figure 12 Observe that the pvalues are well distributed over the complete range and clustered in the center which indicates a good random distribution of the p-values.

\section{G. Final Parameters}

Throughout the paper, we introduced parameters without assigning a definitive value. We now present the configuration we propose for a deployment of BANDANA in real-world applications. The parameters have been carefully chosen based on our evaluations performed on the datasets from Mannheim and Osaka University. When using an accelerometer resolution of $50 \mathrm{~Hz}$, we propose a resampling rate of $\rho=40$ for bit extraction of $b=4$ bits per gait cycle $R_{i}$ resulting in $\tau=$ $\rho / b=10$. We target $M=192$ bit gait fingerprints with a cutoff at $N=128$ bit, i.e., for $b=4$ we extract $q=48$ gait cycles. An upper bound for the required length of the data $r$ is given with $48 \cdot \sim 2 s=96 s$.

Following the results depicted in Figure 10, we chose to parameterize the $\mathrm{BCH}$ codes to allow correction of at maximum $20 \%$ of the bits in the fingerprint with $t=\lfloor 128 \cdot 0.2\rfloor=25$. Consequently, at least $80 \%$ similarity between the fingerprints is required. This results in a 103-bit security level for $\boldsymbol{k}$ used as a password for PAKE. While we have shown that the entropy of our fingerprints is sufficiently high, PAKE takes additional countermeasures for low entropy.

\section{SeCurity Model}

In the following, we analyze BANDANA's security model by discussing possible attack scenarios. The attacks are discussed in order of increasing severity.

1) Mimic Gait: An imposter could try to mimic the gait of the victim to produce fingerprints above $80 \%$ similarity. In BANDANA, the impersonator is constrained to mimic the gait for a very specific time frame where the authentication between the impersonator's device and the victim's device happen. While we do not have actual sensor data of impersonators, the evaluation of the Mannheim dataset show that there are only a small number of accidental collisions between fingerprints from different subjects (cf. Section V-D) over all possible pairwise comparisons of all 14:1 subjects at 10-12 minutes duration each. As shown in [37], a minimal-effort impersonation attack does not improve the chances of success. In BANDANA, the default configuration allows for one try of PAKE only, before starting a completely new authentication process. For $M=192$ bit long fingerprints, BANDANA's full process takes up to $\sim 200 \mathrm{~s}$. Thus, an optimal imposter - one who is always following the victim - is constrained to $\sim 432$ tries per day.

2) Brute Force: Without requiring additional knowledge about the victim's gait, an attacker may want to brute force keys $\boldsymbol{k}$ by exhausting the space of all possible keys $\mathcal{C}=\mathbb{F}_{2^{103}}$. 


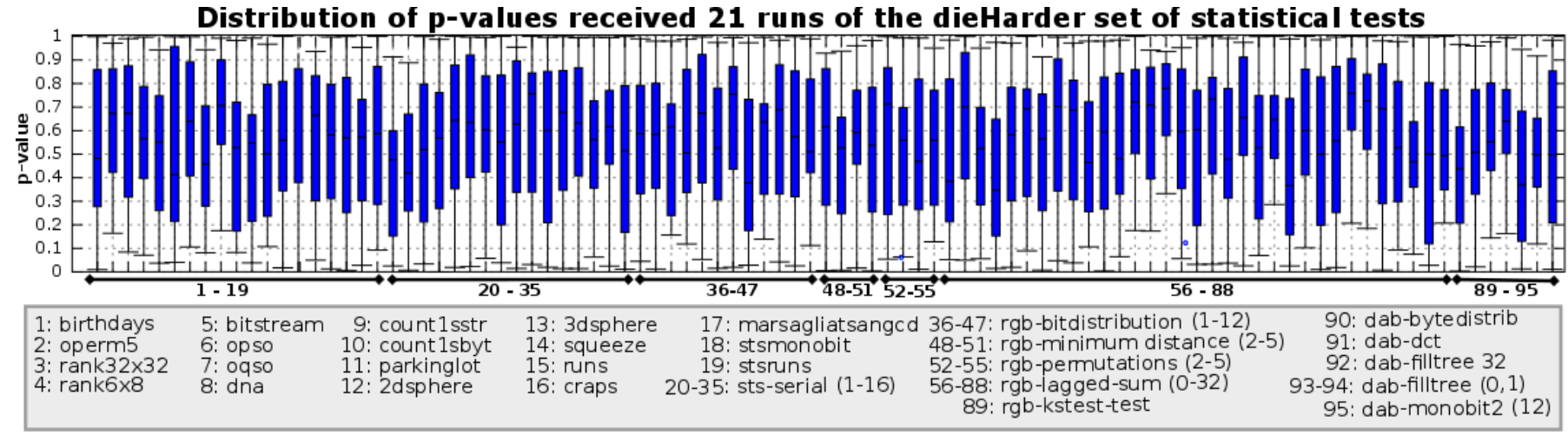

Fig. 12: Distribution of p-values achieved for 128bit keys (fingerprint length $M=192,64$ unreliable bits removed) in the various statistical tests of the dieHarder set of statistical tests.

As discussed in the previous scenario, an attacker is constrained to $\sim 432$ tries per day, where every try generates a new $\boldsymbol{k}$ completely independent from the previous one. Thus, previously tried keys need to be tried again making it impossible to exhaust $\mathcal{C}$.

3) Video Recording: An attacker controlling surveillance cameras could create a video recording of the victim's gait for the timespan where the device-to-device authentication happens. Using motion detection software she could try to infer acceleration values to generate similar fingerprint. It will be difficult to extract all acceleration changes by just extracting features from a video. Recall that our fingerprint is based on tiny convulsions between a sliding window mean gait $\boldsymbol{A}$ and the instantaneous gait cycle $Z_{i}$, happening on the z-axis pointing towards the ground. For every gait cycle $b=4$ bits are extracted from these changes. While we believe that it will be difficult to accomplish this attack, a detailed analysis is left for future work.

4) Attach Malicious Device: To access the BAN, an attacker could attach a malicious device to the body of the victim, e.g. by slipping a small sensor node into the victim's jacket or by selling a compromised device to the victim. This device could create a second communication channel to forward traffic from inside the BAN to an outsider. Due to the fact that BANDANA works without explicit user interaction, this attack could succeed if executed properly and unnoticed. We would like to remark, though, that this physical attack also contains significant risk for the attacker to be revealed when such malicious device is detected.

\section{CONCLUSION}

We have presented BANDANA: the first-ever implicit secure device-to-device authentication scheme for devices worn on the same body. No user interaction is required to establish shared secrets implicitly and authenticated by fingerprints generated from the user's gait. The protocol accounts for errors without comparing the fingerprints directly, but utilizes fuzzy cryptography based on error correcting codes. A novel quantization method for independently generating similar fingerprints at differing sensor positions has been proposed and evaluated. By selecting only reliable bits, we were able to boost the similarity by $4 \%$. Our final fingerprints between devices worn on the same body have a similarity of $82 \%$ in comparison to devices worn on different bodies with $50 \%$. Therefore, we were able to show that BANDANA works considering its security model. Our main future work will be the investigation of the small number of matching fingerprints in the inter-body case and on a detailed entropy analysis.

\section{REFERENCES}

[1] M. Sethi, E. Oat, M. Di Francesco, and T. Aura, "Secure Bootstrapping of Cloud-managed Ubiquitous Displays," in Proceedings of the 2014 ACM International Joint Conference on Pervasive and Ubiquitous Computing, ser. UbiComp '14, Seattle, Washington: ACM, 2014, pp. 739-750.

[2] J. Suomalainen, J. Valkonen, and N Asokan, "Security associations in personal networks: A comparative analysis," in European Workshop on Security in Ad-hoc and Sensor Networks, Springer, 2007, pp. 43-57.

[3] D. Schürmann and S. Sigg, "Secure communication based on ambient audio," Mobile Computing, IEEE Transactions on, vol. 12, no. 2, pp. 358-370, 2013.

[4] S. Sicari, A. Rizzardi, L. A. Grieco, and A. Coen-Porisini, "Security, privacy and trust in Internet of Things: The road ahead," Computer Networks, vol. 76, pp. 146-164, 2015.

[5] M. K. Chong, R. Mayrhofer, and H. Gellersen, "A survey of user interaction for spontaneous device association," $A C M$ Computing Surveys (CSUR), vol. 47, no. 1, p. 8, 2014.

[6] D. Bichler, G. Stromberg, M. Huemer, and M. Löw, "Key generation based on acceleration data of shaking processes," in International Conference on Ubiquitous Computing, Springer, 2007, pp. 304-317.

[7] R. Mayrhofer and H. Gellersen, "Shake well before use: Authentication based on accelerometer data," in Pervasive computing, Springer, 2007, pp. 144-161.

[8] B. Groza and R. Mayrhofer, "SAPHE: simple accelerometer based wireless pairing with heuristic trees," in Proceedings of the 10th International Conference on Advances in Mobile Computing \& Multimedia, ACM, 2012, pp. 161-168.

[9] Y. Liu and J. Niu, "Overlapped-shaking: A local authentication method for mobile applications," in 2014 IEEE Computing, Communications and IT Applications Conference (ComComAp), IEEE, 2014, pp. 93-97. 
[10] R. Mayrhofer, H. Hlavacs, and R. D. Findling, "Optimal derotation of shared acceleration time series by determining relative spatial alignment," International Journal of Pervasive Computing and Communications, vol. 11, no. 4, pp. 454-466, 2015.

[11] R. D. Findling, M. Muaaz, D. Hintze, and R. Mayrhofer, "Shakeunlock: Securely unlock mobile devices by shaking them together," in Proceedings of the 12th International Conference on Advances in Mobile Computing and Multimedia, ACM, 2014, pp. 165-174.

[12] R. D. Findling, M. Muaaz, D. Hintze, and R. Mayrhofer, "ShakeUnlock: Securely Transfer Authentication States Between Mobile Devices," IEEE Transactions on Mobile Computing, vol. PP, no. 99, pp. 1-1, 2016.

[13] T. Van Goethem, W. Scheepers, D. Preuveneers, and W. Joosen, "Accelerometer-Based Device Fingerprinting for Multi-factor Mobile Authentication," in International Symposium on Engineering Secure Software and Systems, Springer, 2016, pp. 106-121.

[14] W. Gu, Z. Yang, L. Shangguan, X. Ji, and Y. Zhao, "ToAuth: Towards Automatic Near Field Authentication for Smartphones," in 2014 IEEE 13th International Conference on Trust, Security and Privacy in Computing and Communications, IEEE, 2014, pp. 229-236.

[15] M. Mehrnezhad, F. Hao, and S. F. Shahandashti, "Tap-Tap and Pay (TTP): Preventing Man-In-The-Middle Attacks in NFC Payment Using Mobile Sensors," in 2nd International Conference on Research in Security Standardisation, 2014.

[16] —, "Tap-Tap and Pay (TTP): Preventing the Mafia Attack in NFC Payment," in International Conference on Research in Security Standardisation, Springer, 2015, pp. 21-39.

[17] R. Mayrhofer, "The candidate key protocol for generating secret shared keys from similar sensor data streams," in European Workshop on Security in Ad-hoc and Sensor Networks, Springer, 2007, pp. 1-15.

[18] R. Jin, L. Shi, K. Zeng, A. Pande, and P. Mohapatra, "MagPairing: Pairing Smartphones in Close Proximity Using Magnetometers," IEEE Transactions on Information Forensics and Security, vol. 11, no. 6, pp. 1306-1320, 2016.

[19] A. Varshavsky, A. Scannell, A. LaMarca, and E. De Lara, "Amigo: Proximity-based authentication of mobile devices," in International Conference on Ubiquitous Computing, Springer, 2007, pp. 253-270.

[20] D. A. Knox and T. Kunz, "Wireless fingerprints inside a wireless sensor network," ACM Transactions on Sensor Networks (TOSN), vol. 11, no. 2, p. 37, 2015.

[21] M. Miettinen, N Asokan, T. D. Nguyen, A.-R. Sadeghi, and M. Sobhani, "Context-based zero-interaction pairing and key evolution for advanced personal devices," in Proceedings of the 2014 ACM SIGSAC Conference on Computer and Communications Security, ACM, 2014, pp. 880-891.

[22] Y. Shu, Y. J. Gu, and J. Chen, "Dynamic authentication with sensory information for the access control systems," IEEE Transactions on Parallel and Distributed Systems, vol. 25, no. 2, pp. 427-436, 2014.

[23] S. Mare, A. M. Markham, C. Cornelius, R. Peterson, and D. Kotz, "ZEBRA: zero-effort bilateral recurring authentication," in 2014 IEEE Symposium on Security and Privacy, IEEE, 2014, pp. 705-720.

[24] C. Cornelius and D. Kotz, "Recognizing whether sensors are on the same body," in International Conference on Pervasive Computing, Springer, 2011, pp. 332-349.

[25] K. Kunze, "Compensating for on-body placement effects in activity recognition," $\mathrm{PhD}$ thesis, Citeseer, 2011.

[26] M. Muaaz and R. Mayrhofer, "Orientation independent cell phone based gait authentication," in Proceedings of the 12th
International Conference on Advances in Mobile Computing and Multimedia, ACM, 2014, pp. 161-164.

[27] T. Hoang, D. Choi, V. Vo, A. Nguyen, and T. Nguyen, "A lightweight gait authentication on mobile phone regardless of installation error," in IFIP International Information Security Conference, Springer, 2013, pp. 83-101.

[28] E. A. Heinz, K. S. Kunze, S. Sulistyo, H. Junker, P. Lukowicz, and G. Tröster, "Experimental evaluation of variations in primary features used for accelerometric context recognition," in European Symposium on Ambient Intelligence, Springer, 2003, pp. 252-263.

[29] M. Muaaz and R. Mayrhofer, "An analysis of different approaches to gait recognition using cell phone based accelerometers," in Proceedings of International Conference on Advances in Mobile Computing \& Multimedia, ACM, 2013, p. 293.

[30] A. Srivastava, J. Gummeson, M. Baker, and K.-H. Kim, "Stepby-step detection of personally collocated mobile devices," in Proceedings of the 16th International Workshop on Mobile Computing Systems and Applications, ACM, 2015, pp. 93-98.

[31] T. Hoang, D. Choi, and T. Nguyen, "Gait authentication on mobile phone using biometric cryptosystem and fuzzy commitment scheme," International Journal of Information Security, vol. 14, no. 6, pp. 549-560, 2015.

[32] T. Hoang, D. Choi, V. Vo, A. Nguyen, and T. Nguyen, "A Lightweight Gait Authentication on Mobile Phone Regardless of Installation Error," in Security and Privacy Protection in Information Processing Systems: 28th IFIP TC 11 International Conference, SEC 2013, Auckland, New Zealand, July 8-10, 2013. Proceedings, L. J. Janczewski, H. B. Wolfe, and S. Shenoi, Eds. Berlin, Heidelberg: Springer Berlin Heidelberg, 2013, pp. 83-101.

[33] A. Juels and M. Wattenberg, "A fuzzy commitment scheme," in Proceedings of the 6th ACM conference on Computer and communications security, ACM, 1999, pp. 28-36.

[34] P. Casale, O. Pujol, and P. Radeva, "Personalization and user verification in wearable systems using biometric walking patterns," Personal and Ubiquitous Computing, vol. 16, no. 5, pp. 563-580, 2012.

[35] T. Hoang, D. Choi, and T. Nguyen, "Gait authentication on mobile phone using biometric cryptosystem and fuzzy commitment scheme," International Journal of Information Security, vol. 14, no. 6, pp. 549-560, 2015.

[36] M. Muaaz and R. Mayrhofer, "Cross Pocket Gait Authentication Using Mobile Phone Based Accelerometer Sensor," in International Conference on Computer Aided Systems Theory, Springer, 2015, pp. 731-738.

[37] D. Gafurov, E. Snekkenes, and P. Bours, "Spoof attacks on gait authentication system," IEEE Transactions on Information Forensics and Security, vol. 2, no. 3, pp. 491-502, 2007.

[38] T. T. Ngo, Y. Makihara, H. Nagahara, Y. Mukaigawa, and Y. Yagi, "The largest inertial sensor-based gait database and performance evaluation of gait-based personal authentication," Pattern Recognition, vol. 47, no. 1, pp. 228-237, 2014.

[39] T. Sztyler and H. Stuckenschmidt, "On-body Localization of Wearable Devices: An Investigation of Position-Aware Activity Recognition," in 2016 IEEE International Conference on Pervasive Computing and Communications (PerCom), (Sydney, Australia, Mar. 14-18, 2016), IEEE Computer Society, 2016, pp. 1-9.

[40] G. Simoneau, "Kinesiology of walking," Kinesiology of the musculoskeletal system: foundations for physical rehabilitation, vol. 1, p. 523, 2002.

[41] M. W. Whittle, "Chapter 2 - Normal gait," in Gait Analysis (Fourth Edition), M. W. Whittle, Ed., Fourth Edition, Edinburgh: Butterworth-Heinemann, 2007, pp. 47-100. 
[42] GSMArena.com, Phone finder results for accelerometer and gyrometer, http : / / www . gsmarena . com / results . php3 ? chkAccelerometer $=$ selected $\&$ chkGyro $=$ selected (accessed on 09/2016), 2016

[43] S. O. Madgwick, A. J. Harrison, and R. Vaidyanathan, "Estimation of IMU and MARG orientation using a gradient descent algorithm," in 2011 IEEE International Conference on Rehabilitation Robotics, IEEE, 2011, pp. 1-7.

[44] F. Hao and P. Ryan, "J-PAKE: Authenticated Key Exchange without PKI," in Transactions on Computational Science XI: Special Issue on Security in Computing, Part II, M. L. Gavrilova, C. J. K. Tan, and E. D. Moreno, Eds. Berlin, Heidelberg: Springer Berlin Heidelberg, 2010, pp. 192-206.

[45] T. D. Wu et al., "The Secure Remote Password Protocol," in NDSS, vol. 98, 1998, pp. 97-111.

[46] J. Lester, B. Hannaford, and G. Borriello, ““'Are You with Me?"-Using Accelerometers to Determine If Two Devices Are Carried by the Same Person," in International Conference on Pervasive Computing, Springer, 2004, pp. 33-50.

[47] R. G. Brown, Dieharder: A Random Number Test Suite, http: //www.phy.duke.edu/ rgb/General/dieharder.php 2011. 